\title{
EDISON:
}

\section{A SECOND GENERATION INFRARED SPACE OBSERVATORY}

\author{
H. A. THRONSON, JR. \\ Royal Observatory, Edinburgh and Wyoming Infrared Observatory, \\ University of Wyoming \\ T. G. HAWARDEN \\ Royal Observatory, Edinburgh and Joint Astronomy Centre, Hilo \\ and \\ C. M. MOUNTAIN, J. K. DAVIES, T. J. LEE, AND M. LONGAIR \\ Royal Observatory, Edinburgh
}

\begin{abstract}
EDISON is a large-aperture telescope under study for the second generation of infrared space observatories, whether in orbit or on the moon. The optics equilibrate via radiative cooling to temperatures between 40 and $80 \mathrm{~K}$, depending upon, for example, telescope structure and location. At these temperatures, telescope emission is below that of the astronomical background at all wavelengths shortward of $20-40 \mu \mathrm{m}$. The detector components can be cooled via mechanical refrigerators now in an advanced stage of development. A mixture of radiative and mechanical cooling means that there is no natural limit to EDISON's lifetime. In addition, the upper stage rocket fairing can be almost filled with light-collecting optics and alternative low-emissivity optical designs, such as off-axis systems, can be easily engineered. We are presently evaluating a design for a $2.5 \mathrm{~m}$ observatory to be launched in collaboration with European astronomers as part of the NASA Explorer program. In this presentation, we describe possible spectroscopic and spectrophotometric studies of very faint infrared sources that will require large-aperture space telescopes working at the celestial background limit.
\end{abstract}

\section{Program Summary}

A class of Passively-cooled Orbiting InfraRed Observatory Telescopes (POIROTs) are being studied for second-generation infrared space observatories, both in orbit and on the moon. These are long-lived, large-aperture facilities, using a mixture of radiative and mechanical cooling of optics and detector elements. In our current models, the telescope optics equilibrate at between 40 and $80 \mathrm{~K}$, depending upon radiator design, orbit selection, role of mechanical cooler, and heat load from the electronics. Although POIROTs are much warmer than cryogenic telescopes, radiation from the telescope is less than that from the natural (astronomical) background at all wavelengths shortward of 20-40 $\mu \mathrm{m}$. Unencumbered by a toroidal cryogen tank, an upper stage fairing can be nearly filled with the light-collecting optics of a radiatively-cooled observatory.

Our current proposal is for EDISON ${ }^{1}$ a $2.5 \mathrm{~m}$ POIROT-type telescope, designed to match the capacity of the presently-available large Atlas shroud. Freeing the

1 Thomas A. Edison was probably the premier infrared astronomer of the 19th century. From a henhouse near Rawlins, Wyoming in July, 1878, he detected the near-infrared emission from both $\alpha$ Boo and the solar corona.

Y. Kondo (ed.), Observatories in Earth Orbit and Beyond, 501-505.

(C) 1990 Kluwer Academic Publishers. Printed in The Netherlands. 


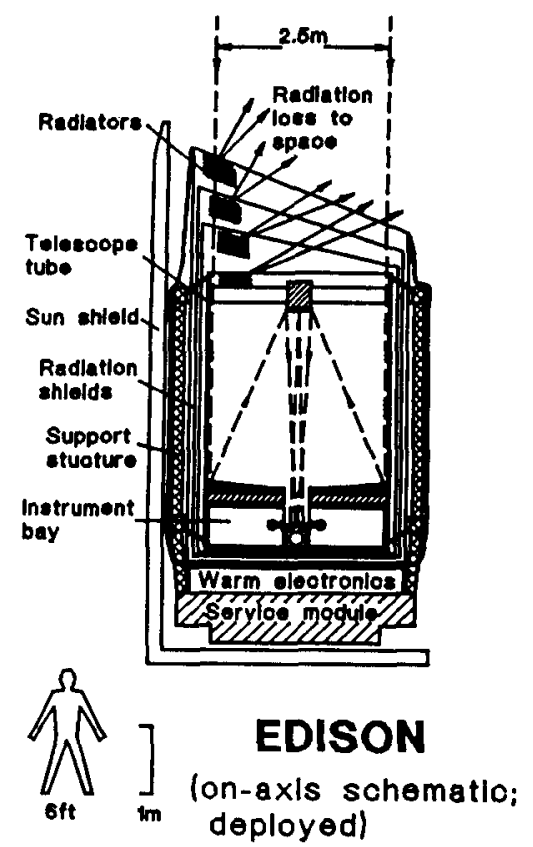

Fig. 1. Schematic design of EDISON as deployed in an on-axis configuration. Significant improvement in performance should be realized with an off-axis design.

telescope from cryogen tanks allows us to explore alternative designs, such as verylow-emissivity and off-axis optics, to further reduce contaminating emission while maintaining a large aperture. We are also studying the feasibility of even larger monolithic telescopes, such as a $\sim 3.3 \mathrm{~m}$ EDISON launched by a Titan IV rocket.

As described in the following pages, a large aperture, in addition to a very long lifetime, will allow EDISON to, for example, [1] deeply survey for brown dwarfs, protogalaxies, protostars, and circumstellar matter; [2] study solid state and gaseous features in objects as diverse as Jovian planets and high- $z$ galaxies; [3] image such objects as circumstellar disks and extragalactic circumnuclear material at arcsecond resolution or better in the near- and mid-infrared; and [4] easily search for nearby sub-stellar and planet-like objects, and undertake a program of classification of their atmospheres.

EDISON is being proposed as a joint American-European venture, launched as part of the NASA Explorer program.

\section{EDISON Design}

\subsection{Radiative Cooling}

Unheated instruments in space naturally cool to temperatures well below that of the same structure on the surface of the earth. For example, the IRAS satellite telescope, 


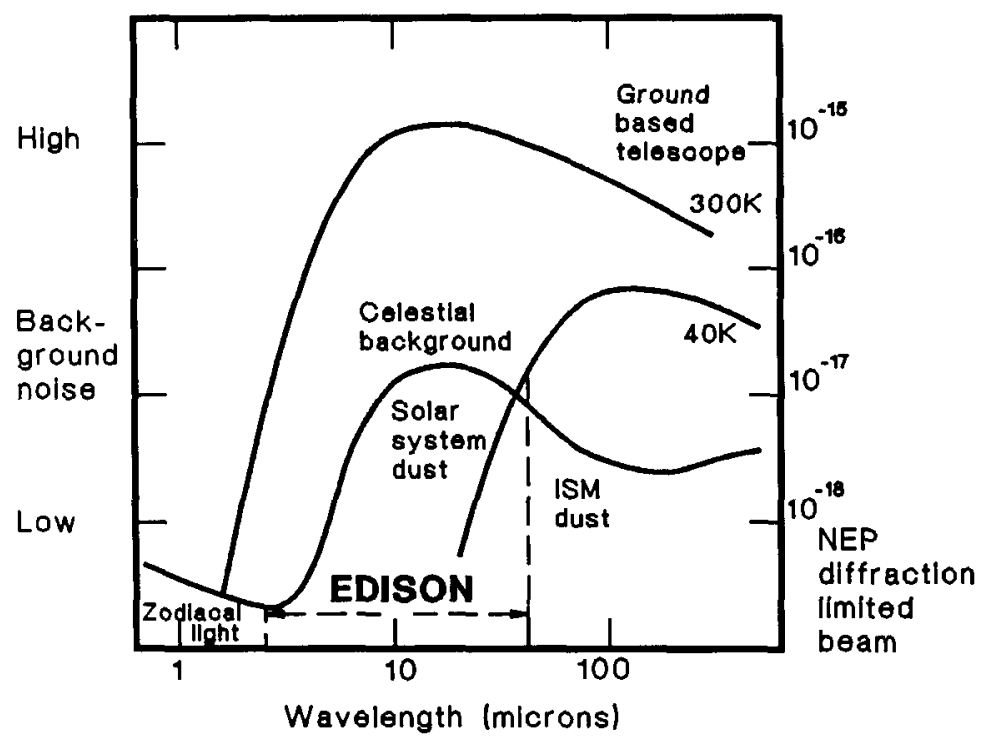

Fig. 2. The limiting sensitivity of an infrared telescope in space is determined in a large measure by the radiation on the detectors. Here we show the thermal emission from our $40 \mathrm{~K}$ EDISON model and a warm groundbased telescope, compared to the celestial background in space.

which was not optimized for radiative cooling, equilibrated at about $100 \mathrm{~K}$ after cryogen boiloff. Large-aperture infrared telescopes which are specifically designed to be cooled via radiation should, therefore, reach background-limited performance throughout the near- and much of the mid-infrared. A schematic drawing of one design for EDISON is shown in Figure 1. In this particular model, a deployable or inflatable sun shield provides significant shading and the set of three nested shields and radiators dominates the radiative cooling.

One preliminary thermal model for EDISON equilibrates at a telescope temperature of roughly $40 \mathrm{~K}$. As shown in Figure 2, radiation from optics at this temperature is below that of the celestial background at wavelengths shortward of about $30 \mu \mathrm{m}$. As the figure demonstrates, in addition to atmospheric extinction and radiation throughout the infrared, emission from the telescope is a major limitation to observations of faint infrared sources from the earth's surface.

\subsection{Closed-Cycle Refrigerators}

Although some possible detector components operate well at the temperatures achievable by radiative cooling alone, long-lived closed-cycle coolers will be necessary to achieve maximum performance. Temperatures as low as $\sim 4 \mathrm{~K}$ are the 


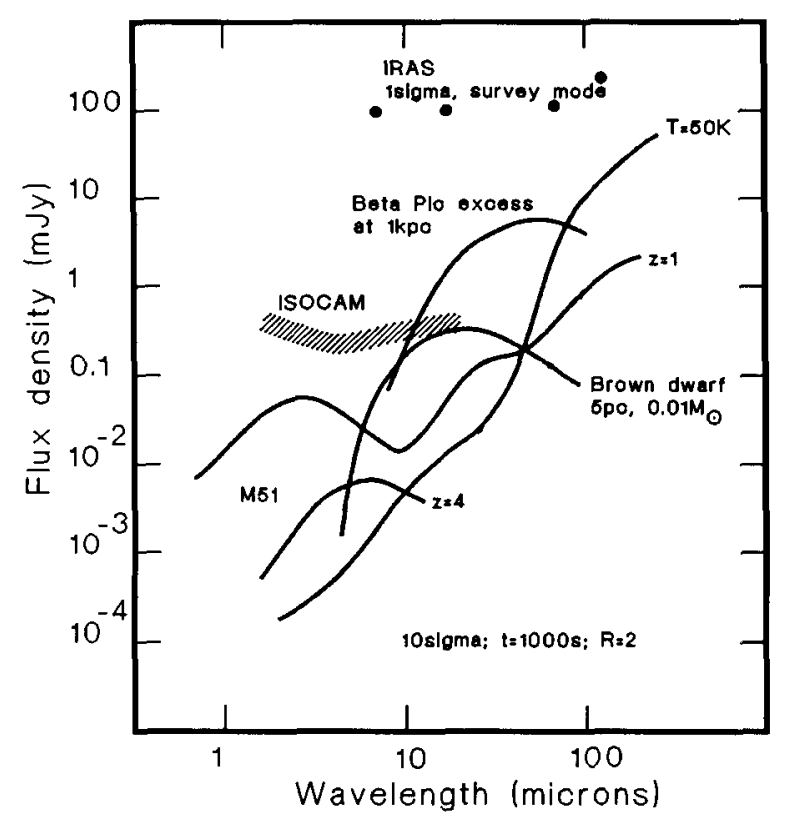

Fig. 3. High-sensitivity photometry with EDISON based on a telescope equilibrium temperature of $50 \mathrm{~K}$.

goal of the three-stage Joule-Thomson/Stirling cycle refrigerators now in an advanced stage of development by Rutherford Appleton Laboratory under contract to the European Space Agency.

\section{EDISON Scientific Capability}

\subsection{Photometric Programs}

Figure 3 demonstrates the sensitivity of a proposed broadband camera using infrared arrays with detector capabilities expected for the early- to mid-1990's. Also shown are a variety of astronomically interesting objects, as well as the performance of IRAS in survey mode and that expected for ISOCAM. Note that nearly-normal galaxies such as M51 can be investigated at high $z$, allowing EDISON to study, for example, evolution of stellar populations using broadband photometry. The high angular resolution of EDISON will be necessary in the confusion-limited environment of galaxies at large redshift. This sub-arcsecond resolution at wavelengths shortward of about $10 \mu \mathrm{m}$ will also be useful in studying "Vega"-like circumstellar material around stars at great distances within the Milky Way.

\subsection{Spectrophotometric and Spectroscopic Programs}

A major justification for infrared space observatories is their ability to obtain spectra of galaxies at high redshift. EDISON will be able to obtain spectra of infrared 


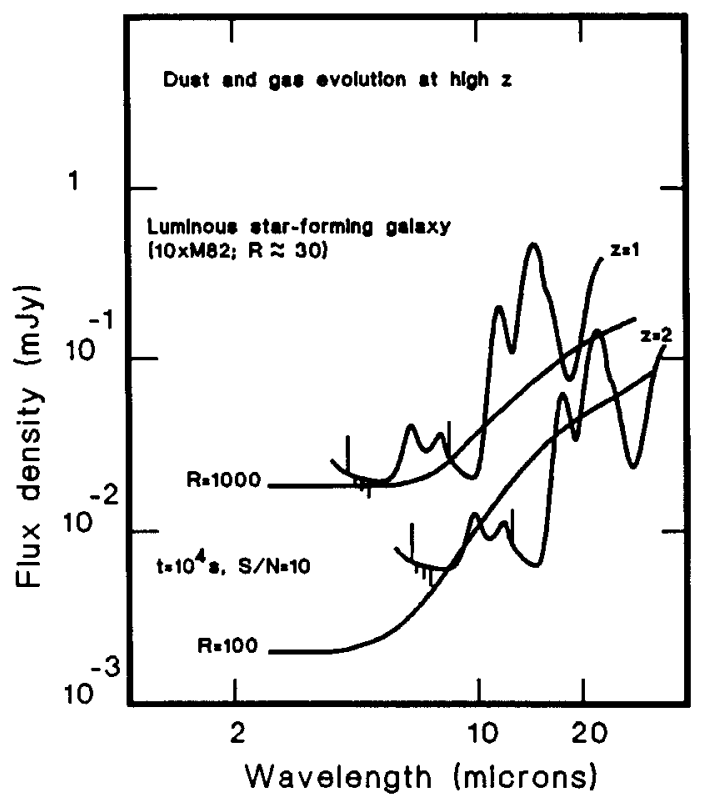

Fig. 4. Spectrophotometry of the near-infrared gas and dust features in high- $z$ galaxies can be obtained by EDISON to investigate the evolution of the ISM. The model spectra show the near- and mid-infrared dust features for an object 10 times more luminous than M82. Also shown are the positions of the hydrogen $\operatorname{Br} \alpha$ and $\operatorname{Br} \gamma$ lines, plus the first overtone $\mathrm{CO}$ absorption band.

gas and dust features after modest integration times, again taking advantage of its high angular resolution to lower the confusion limit for distant objects. One program of particular importance, as illustrated in Figure 4, will be study of the evolution of interstellar gas and dust features via intermediate-resolution spectra of very distant galaxies as a function of redshift.

A decade from now, many candidate brown dwarfs and other sub-stellar planetlike objects should have been identified and the essential task of classification will be carried on with high-sensitivity infrared spectroscopy. Our current proposal for a $2.5 \mathrm{~m}$ EDISON will be sufficiently sensitive to not only be used for study of the atmospheres of brown dwarfs, but hypothetical Jovian and sub-Jovian objects will also be investigated. As an example of performance, the earth could be clearly identified as a non-Jovian planet at a distance of about $5 \mathrm{pc}$ after an integration time of only 2 days. The major limitation to a campaign of planetary classification using EDISON is not sensitivity, but the extreme difficulty of discrimination between the target and a neighboring star. On the other hand, it is reasonable to suppose that many sub-stellar, planet-like objects exist either at great distances from a central star or entirely isolated. Furthermore, EDISON should be considered as the prototype for one element of an interferometer or spatial array that would have the capability to discriminate between a star and surrounding planets. 